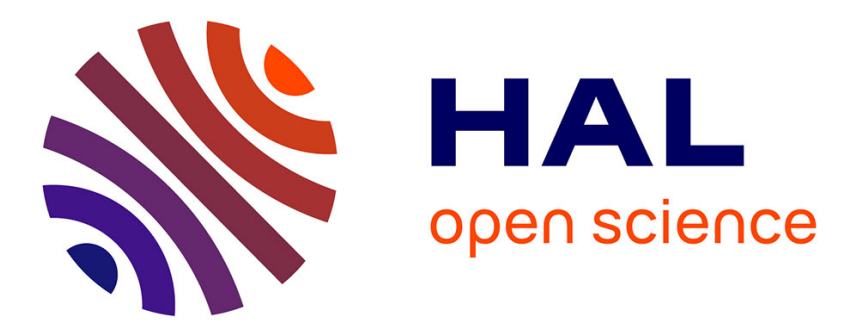

\title{
Une critique d'explication par les causes finales: l'anticontractualisme de Hume. Une histoire naturelle du politique
}

Céline Bonicco-Donato

\section{To cite this version:}

Céline Bonicco-Donato. Une critique d'explication par les causes finales: l'anticontractualisme de Hume. Une histoire naturelle du politique. Dialogue: Canadian Philosophical Review / Revue canadienne de philosophie, 2007, 46, pp.637-662. 10.1017/S0012217300002158 . hal-01525429

\author{
HAL Id: hal-01525429 \\ https://hal.science/hal-01525429
}

Submitted on 20 May 2017

HAL is a multi-disciplinary open access archive for the deposit and dissemination of scientific research documents, whether they are published or not. The documents may come from teaching and research institutions in France or abroad, or from public or private research centers.
L'archive ouverte pluridisciplinaire HAL, est destinée au dépôt et à la diffusion de documents scientifiques de niveau recherche, publiés ou non, émanant des établissements d'enseignement et de recherche français ou étrangers, des laboratoires publics ou privés.

\section{(1) (1) $\$$}

Distributed under a Creative Commons Attribution - NonCommercial - NoDerivatives $\mid 4.0$ 


\title{
Une critique d'explication par les causes finales :
}

\section{l'anticontractualisme de Hume}

\author{
Une histoire naturelle du politique
}

\section{Céline Bonicco Paris 1-Fondation Thiers}

La critique du paradigme dominant de la philosophie politique du XVIII ${ }^{\text {ème }}$ siècle, le contractualisme, constitue le versant le plus fameux de la philosophie politique de Hume. Le philosophe n'a cessé de dialoguer, explicitement ou implicitement, avec Hobbes et Locke tout au long de son oeuvre. La critique humienne est aussi connue que décriée. Cependant, elle est moins appréhendée en elle-même que par son utilisation par Burke ${ }^{\mathrm{ii}}$ pour porter des coups de butoir à la Révolution française et aux Droits de l'homme. De l'anticontractualisme à la pensée contre-révolutionnaire, la conséquence semble être bonne. Un tel héritage sulfureux a suscité une double analyse de l'analyse humienne.

- Dans la première, il s'agit de sauver Hume de lui-même. Deux possibilités se présentent alors. Montrer que loin de rompre avec le contractualisme, il ne fait que l'étirer et l'étaler dans le tempsiii, ou montrer que la convention qu'il prétend substituer au contrat, n'est que le genre dont le contrat est une espèce ${ }^{\mathrm{iv}}$.

- Dans la deuxième, il s'agit de discréditer la pertinence philosophique de l'analyse de Hume en mettant au jour les prémisses idéologiques sous-jacentes ${ }^{v}$.

Dans le cadre de cet article, nous nous proposons d'analyser la critique humienne du contractualisme indépendamment de ses répercussions sur la pensée contrerévolutionnaire, pour l'envisager comme une analyse philosophique cohérente, sans que 
l'interprétation soit faussée par son héritage. Il s'agit ainsi de comprendre comment elle tire les conséquences politiques d'analyses internes à sa propre philosophie. Nous laissons donc de côté la question de savoir si la présentation humienne du contractualisme rend justice aux textes de Hobbes et de Locke, en indiquant simplement que si la pensée du premier est le plus souvent restituée de manière exacte, ce n'est pas le cas pour celle du second ${ }^{\mathrm{vi}}$. Hume vise avant tout, comme en témoigne la lettre du 13 février $1748^{\text {vii }}$, la vulgate lockienne sur laquelle repose l'idéologie Whig, ce qui explique un certain nombre d'approximations. Par cette lecture immanente à l'œuvre du philosophe écossais, nous voudrions mettre à l'épreuve l'hypothèse suivante : la critique humienne du contractualisme est une critique de l'application au domaine politique de l'argument du dessein présenté dans les Dialogues sur la religion naturelle ${ }^{\text {viii }}$. Elle constitue le pendant dans le monde des affaires humaines de la mise à mal du principe de raison dans le cadre général de l'univers. Rien d'étonnant à cette symétrie puisque l'homme n'est pas un empire dans un empire : la nature humaine fait bel et bien partie de la nature. Le contractualisme en rendant compte de l'ordre social par un accord libre et volontaire passé entre les individus en vue de leur bien-être, ne fait que réemployer dans le monde de la pratique l'argument du dessein déployé par les théistes pour rendre compte de la nature. Il explique la réalité politique par un principe hétérogène, un fondement : l'instauration de la société correspondrait à un dessein des individus de l'état de nature, à un projet réalisé volontairement répondant à une fin fixée par la raison. Hume ne rejetterait pas tant le contractualisme pour des raisons politiques que pour des raisons méthodologiques : une explication par les causes finales, quel que soit le domaine concerné, n'est jamais une explication satisfaisante. Nous ne voulons pas dire par là que les causes finales ne jouent aucun rôle dans les comportements humains, puisqu'il est manifeste que les hommes poursuivent des buts, mais qu'en dépit de cela, une explication de type finaliste n'est pas recevable dans la mesure où elle conduit inévitablement à introduire des causes finales 
fictives. Hume critiquerait donc l'hypothèse contractualiste pour le rôle de fondement qu'elle fait jouer au contrat et pour la psychologie téléologique qu'elle attribue aux acteurs sociaux.

Certes, la rédaction des Dialogues est postérieure de trois ans à la publication de l'essai « Du contrat originel » et de quatorze ans à celle du livre 3 du Traité de la nature humaine. Mais si cet ouvrage peut éclairer des analyses antérieures de Hume, c'est dans la mesure où, loin de marquer une rupture dans sa philosophie, il constitue au contraire l'achèvement de sa théorie de la causalité. La maturité philosophique alors atteinte par Hume se révèle extrêmement féconde pour lire au fil de son épistémologie ses analyses morales et politiques.

Aborder de cette manière la critique humienne du contractualisme permet de considérer sa théorie politique alternative comme l'application d'un mode explicatif non finaliste. La convention ${ }^{\mathrm{ix}}$ est le concept qui permet de penser dans le monde politique l'ordre sans la finalité selon les préceptes épistémologiques des Dialogues sur la religion naturelle. La société est une forme particulière d'ordre, mais elle s'engendre sans raison. Dans « $\mathrm{Du}$ contrat originel », «L'origine du gouvernement $»^{\mathrm{x}}$, et le livre 3 du Traité de la nature humaine se déploie une généalogie du politique dans le cadre d'une causalité naturelle, où la sympathie comme principe remarquable de la nature humaine se substitue au principe de raison.

Cette présentation de la critique humienne dans sa dimension négative et positive soulève néanmoins une objection quant à sa pertinence. La réfutation humienne ne raterait-telle pas sa cible en se bornant à montrer que le contrat ne peut être l'origine causale des sociétés ? En effet, le contractualisme ne prétend pas montrer que le contrat est l'origine des sociétés existantes, mais qu'il doit l'être. Son propos n'est pas historique mais avant tout normatif : seul le contrat peut fonder une autorité légitime. La critique de Hume serait-elle alors décalée par rapport à son objet ? Absolument pas. Deux arguments, l'un faible, l'autre 
fort, permettent de lever cette objection. Hume, en effet, vise l'idéologie Whig qui se réfère à l'ancienne constitution saxonne comme origine historique du gouvernement pour contester la dynastie des Stuart ${ }^{\mathrm{xi}}$. Ainsi la discussion humienne ne témoigne-t-elle pas d'une méconnaissance de la théorie contractualiste mais doit être éclairée par la forme que celle-ci revêtait dans l'Angleterre de la seconde moitié du XVIII ${ }^{\text {ème }}$ siècle. Mais de manière plus intéressante pour la portée générale de sa critique, Hume ne s'en tient pas là. Il déboute le contrat non seulement comme origine causale historique mais également comme fondement possible des sociétés. Les deux points sont intrinsèquement liés dans l'essai «Du contrat originel » ${ }^{\mathrm{xii}}$, et les Dialogues sur la religion naturelle permettent de comprendre l'articulation entre l'origine-événement et l'origine-fondement, en leur substituant une notion d'origine comprise comme déploiement d'un principe. Non seulement, le contrat n'est pas la cause des sociétés, mais il ne peut les fonder dans la mesure où la légitimité n'est jamais a priori mais toujours le fruit d'une construction. Penser ainsi la légitimité n'est pas faire profession de relativisme mais s'efforcer d'inscrire le normatif au sein d'une histoire naturelle.

Nous examinerons tout d'abord comment la mise en évidence de la faiblesse épistémologique du recours aux causes finales constitue le cadre d'interprétation pertinent de la critique humienne du contractualisme. La genèse positive du politique déployée par Hume sera ensuite envisagée comme application particulière du seul mode d'explication pertinent des phénomènes, l'histoire naturelle.

\section{Le paradigme contractualiste comme formulation politique de}

\section{l'argument du dessein}

Les Dialogues sur la religion naturelle sont une machine de guerre contre l'argument du dessein de la théologie rationnelle. Cet argument pris en charge par le porte- 
parole de cette dernière, Cléanthe, va être soumis au travail de sape de l'argumentation du sceptique Philon.

Pour prouver l'existence de Dieu et identifier ses attributs, Cléanthe met en place une analogie s'appuyant sur l'expérience. Son raisonnement vise l'affirmation du fidéiste Déméa, malicieusement relayée par Philon, selon laquelle la nature divine est inconnaissable ${ }^{\text {xiii }}$. Le point de départ de son raisonnement est la constatation d'une ressemblance entre le monde et les objets artificiels. L'observation révèle qu'il est comme une grande machine divisée en une infinité de machines plus petites, ajustées les unes aux autres. Il s'agit d'un mécanisme puissant et complexe dont la différence avec les machines créées par l'homme n'est que quantitative. Le monde présente un ajustement des moyens aux fins: chaque partie est ordonnée et proportionnée pour répondre à une fonction précise. Or l'expérience nous a appris que des effets semblables sont produits par des causes semblables. Puisque les objets artificiels auxquels le monde ressemble sont produits par l'intelligence humaine, l'on doit nécessairement conclure qu'une volonté intentionnelle les a conçus et créés pour répondre à une fin. Une inférence naturelle s'impose alors, le monde a été produit par l'intelligence divine de la même manière qu'une machine est créée par l'esprit de l'homme ${ }^{\mathrm{xiv}}$.

Or, l'argument contractualiste critiqué par Hume dans l'essai « Du contrat originel », peut apparaître comme une politisation de cet argument du dessein. Ici, la société est en effet pensée comme un produit artificiel, comme une machine créée par une volonté intentionnelle et productrice visant une certaine fin. Si l'entrée en société apparaît comme un sacrifice par les individus d'une partie de leur liberté, il importe de préciser qu'il s'agit d'un sacrifice volontairement consenti, de telle sorte que dans les deux cas, le dessein de Dieu et celui des individus, il y a bien exercice de la liberté. Un écart entre l'ordre du monde et l'ordre social doit cependant être noté. Alors que le premier est l'expression d'une volonté 
unique, la volonté divine, le second est l'expression d'une multiplicité de volontés, la volonté des individus de l'état de nature. Néanmoins, il faut immédiatement préciser que dans les théories contractualistes, chez Hobbes comme chez Locke, ces volontés sont unifiées dans leur contenu. Le contrat repose sur un accord entre les volontés, sur leur convergence vers une même fin.

Il convient donc d'analyser ce qui dans l'analyse de Hume autorise ce rapprochement entre contractualisme et théologie naturelle : quel cadre téléologique mettent en place Locke et Hobbes pour penser la société comme l'effet d'une intention rationnelle? en quoi le contractualisme peut-il alors apparaître comme une religion politique ? Pourquoi l'analyse de la causalité conduit-elle Hume à rejeter la pertinence de l'explication par les causes finales?

\section{- La finalité à l'œuvre dans les théories contractualistes}

L'argument du dessein présenté par les partisans de la théologie naturelle pour inférer l'existence de Dieu à partir de l'observation de la perfection du monde est un argument de type finaliste, la finalité étant entendue comme la réalisation d'une intention. Nous constatons dans les choses ordre et harmonie, et puisque la cause de cet ordre et de cette harmonie n'est pas dans les choses mêmes et que le hasard ne peut rendre compte de cette perfection, il faut conclure à l'existence d'un sage ordonnateur créant à partir de la représentation de fins. Le monde a pour ainsi principe une causalité qui est intelligence et volonté.

Ce qui va motiver la critique humienne des théories contractualistes de Hobbes et de Locke, est la persistance d'un tel cadre téléologique dans leurs analyses politiques. L'introduction implicite de la finalité à partir de prémisses psychologiques 
anthropologiquement fausses commande son rejet de cette théorie et autorise à le rapprocher de la critique qu'il fait de l'argument du dessein.

Hume, en effet, critique vertement l'hypothèse égoïste des deux auteurs dans l'Enquête sur les principes de la morale ${ }^{\mathrm{xv}}$, or lorsqu'il examine la genèse du politique, il présente cette hypothèse égoïste comme une hypothèse erronée, reposant sur une méconnaissance de la nature humaine ${ }^{\mathrm{xvi}}$. Pourquoi prend-il la peine d'examiner cette hypothèse dans le cadre d'un examen politique ? Dans la mesure où, au sein du cadre téléologique du contractualisme, anthropologie et politique sont indissociables : si l'égoïsme constitue la fin pratique guidant les actions des hommes et si la représentation de la satisfaction individuelle par l'entendement éclaire notre volonté, cela reste vrai lorsque l'institution de la société est en jeu. C'est par la considération de l'homme comme égoüste, erreur anthropologique et psychologique, que s'introduit le finalisme dans l'explication du politique.

Ainsi la séquence, raison-volonté-action joue-t-elle à plein régime lors du contrat social. Les hommes vivant dans l'état de nature se rendent compte rationnellement que leur vie est menacée s'ils continuent à vivre comme cela ; visant à satisfaire l'amour de soi, ils cherchent un moyen artificiel pour remédier à la situation dans laquelle ils sont. La volonté éclairée par la représentation de cette fin, se porte sur la société. Le contrat social est le moyen mis en place pour satisfaire l'égoïsme. Puisque la société apparaît comme le produit artificiel d'une intention raisonnable, elle est une forme d'ordre dont on peut rendre raison par le recours à une intention extérieure. Hobbes emploie expressément le terme de cause finale dans le chapitre XVII du Leviathan ${ }^{\text {xvii }}$. Cette transposition de l'argument du dessein sur le plan politique n’a pas échappé aux commentateurs des théories contractualistes qui ont souligné dans la mise en place du pacte social chez Hobbes, comme chez Locke, la présence de « l'argument du fabricant » : la relation entre un ouvrier et son œuvre est prise comme modèle 
non seulement pour comprendre la création du monde, mais aussi la création du politique. Il s'agit d'un «artifice délibéré qui, en toute connaissance de cause, pour réaliser des fins déterminées, fait apparaître une réalité nouvelle durable » ${ }^{\text {xiii }}$.

La critique humienne du contractualisme ne repose donc pas sur des bases idéologiques, la sympathie supposée de Hume pour le thorisme, mais est commandée par une raison épistémologique, l'intervention dans leur argumentation d'une explication par les causes finales. C'est dans la mesure où, les contractualistes rendent compte d'un phénomène apparemment ordonné par le recours à une volonté intentionnelle raisonnable, de la même manière que peuvent le faire les partisans de la théologie naturelle quand le monde est en cause, que Hume critique ces deux théories. La critique de l'argument du dessein et la critique du contractualisme prennent place dans la critique générale du finalisme. Si l'inscription de la critique de la théologie naturelle dans ce cadre est manifeste dans la section XI de l'Enquête sur l'entendement humain ${ }^{\text {xix }}$, cela est également vrai pour le contractualisme comme le montre le détail de l'analyse humienne où le philosophe invalide l'intervention d'une intention raisonnable.

S'il faut «considérer l'hypothèse religieuse uniquement comme une méthode particulière d'explication des phénomènes visibles de l'univers $»^{\mathrm{xx}}$, il faut considérer l'hypothèse contractualiste uniquement comme une méthode particulière d'explication des phénomènes visibles de la pratique humaine, les phénomènes politiques existants ou à venir.

\section{- Le contractualisme comme enthousiasme politique}

Le rapprochement opéré par Hume entre le raisonnement des contractualistes et celui des partisans de la théologie naturelle est explicite dans l'essai « Du contrat originel » ${ }^{\mathrm{xxi}}$ : le philosophe met sur le même plan ceux qui font résider l'origine du gouvernement dans la 
divinité et ceux qui la font résider dans le contrat originel. Dans les deux cas, il y intervention d'une finalité rationnelle, intentionnelle et productrice : un Être ou des êtres se représentent intellectuellement une fin (le bien de l'humanité ou le bien individuel) qui met en branle leur volonté et aboutit à la production d'un effet. Dans les deux cas, on explique une forme d'ordre par une cause transcendante au phénomène à expliquer.

De cette manière devient pleinement compréhensible le lien établi par Hume entre le contractualisme et l'enthousiasme ${ }^{\mathrm{xxii}}$ : si cette théorie politique apparaît comme une « humanisation » de l'argument du dessein, on peut aussi dire parallèlement qu'elle est une « divinisation » de l'homme, se substituant dans le champ politique au Créateur. La jonction entre enthousiasme et contractualisme se fait sous le chef de l'exaltation de l'individu et de la méconnaissance des médiations.

Comment Hume présente-t-il l'enthousiasme?

L'enthousiasme est une corruption de la vraie religion. Un esprit enthousiaste est sujet à je ne sais quelle élévation prétentieuse qui naît d'un heureux succès, d'une santé prospère, d'un naturel ardent, ou d'une disposition hardie et confiante. Dans un tel état d'esprit, l'imagination s'enfle et se remplit de grandes mais confuses conceptions, qu'aucune beauté ni aucune joie sublunaire ne sauraient égaler ${ }^{\text {xiii. }}$.

L'enthousiaste se caractérise par un excès de confiance: il s'affirme comme origine absolue en méconnaissant les intermédiaires qui sont nécessaires à son affirmation.

Libre champ est laissé à la fantaisie qui parcourt les régions invisibles ou le monde des esprits, lieux où l'âme peut se livrer sans retenue aux chimères les plus conformes à son goût et à sa disposition du moment ${ }^{\text {xiv }}$. 
Le contractualiste fait, lui aussi, preuve d'hybris. Il nie toute médiation entre l'individu et lui-même et méconnaît sa faiblesse intrinsèque et le rôle que joue la société dans la construction de son identité. Hume présente les caractéristiques de l'individu impliqué dans les théories contractualistes comme relevant d' "un état de perfection bien au-dessus de la condition humaine ${ }^{\mathrm{xxv}}$. Le contractualiste présente toutes les qualités qui sont à la source de l'enthousiasme : «l'espoir, la suffisance, la présomption, une imagination échauffée, jointes à l'ignorance ${ }^{\mathrm{xxvi}}$. On peut relever quatre traits distinctifs de ce fanatisme religieux et de son expression politique : autonomie personnelle, droits individuels, absence de médiation entre l'homme et Dieu, constructions dérivées des phénomènes moraux et politiques d'après nos propres lumières $^{\mathrm{xxvii}}$.

Le contractualisme apparaît bien comme « le credo d'(un) parti, dont les membres s'enorgueillissent avec raison de la solidité de leur philosophie et de leur liberté de pensée ${ }^{\text {xxviii }}$, et conduit à une exaltation dangereuse.

La fureur actuelle du peuple, bien que dissimulée sous des prétentions à la liberté civile, est en réalité excitée par le fanatisme religieux, un des principes les plus aveugles, obstinés et ingouvernables qui puissent mouvoir la nature humaine ${ }^{\mathrm{xxix}}$.

\section{- L'invalidation de l'argument du dessein}

Hume attire ainsi l'attention du lecteur sur le cadre téléologique du contractualisme par la mise en évidence de son versant anthropologique et psychologique, la considération de l'homme comme égoïste. Cependant, il ne suffit pas de dire que cette doctrine se présente comme une politisation de l'argument du dessein pour comprendre sa critique. Encore faut-il préciser pourquoi l'analyse de la causalité déployée tant dans l'Enquête sur l'entendement humain, que dans le livre 1 du Traité de la nature humaine, le conduit à rejeter l'argument du dessein et à proposer un autre modèle épistémologique. Nous 
nous intéresserons moins à l'invalidation bien connue de l'explication par les causes finales qu'au type substitutif d'explication mis en place pour rendre compte des phénomènes ordonnés. Il ne s'agit pas pour Hume de renoncer à expliquer l'ordre, mais de l'expliquer en le dissociant de toute perspective finaliste. La méthode positive mise en place est d'une importance capitale dans la mesure où elle éclaire la genèse du politique décrite dans le livre 3 du Traité de la nature humaine.

Philon mène une double réfutation de l'argument du dessein dans les Dialogues sur la religion naturelle, négative dans la deuxième partie, et positive dans la quatrième partie où il met en place une méthode substitutive. Il faut noter une gradation dans cette analyse : en effet, si la réfutation négative critique moins la dimension finaliste de l'argument du dessein que la disproportion entre l'effet observé et sa cause, en considérant la causalité du dessein comme un mode de causalité parmi d'autres sans s'interroger sur sa spécificité, la réfutation positive critique de manière explicite le recours à l'intention. Autrement dit, si l'angle d'attaque de la première critique consiste à battre en brèche la pertinence du principe de raison à partir de l'examen sceptique du principe de causalité, le second le conteste en tant que tel. Ces deux critiques permettent d'éclairer les deux moments traditionnellement distingués dans «Du contrat originel»: le premier invalidant le contrat comme origine causale, et le second comme fondement possible.

Dans la deuxième partie des Dialogues sur la religion naturelle, Philon rejette l'argument du dessein par une réflexion sur l'analogie qui le sous-tend. Cléanthe invoque l'argument expérimental bien connu, suivant lequel, si l'on a expérimenté la conjonction entre une cause et un effet, on peut inférer à coup sûr qu'un effet semblable est causé par le même phénomène que l'est l'effet auquel il ressemble. Philon ne conteste absolument pas que 
l'attribution d'une cause semblable à un effet semblable ne soit une inférence expérimentalement valide. Ce qu'il met en cause est la réduction de l'argument du dessein à un tel raisonnement causal : la ressemblance entre l'univers et un produit manufacturé n'est pas suffisamment grande pour que l'on puisse dire que ce sont deux effets semblables et donc pour que l'on puisse inférer qu'ils ont la même cause, une intention raisonnable.

(...) Vous n'affirmerez sûrement pas que l'univers entretient avec une maison une ressemblance telle que nous puissions avec la même certitude inférer une cause semblable, ni que l'analogie soit entière et parfaite. La dissimilitude est si frappante que tout ce à quoi vous pouvez prétendre se monte à une supposition, une conjecture, une présomption, concernant une cause semblable ; et comment cette prétention sera reçue dans le monde, je vous le laisse envisager ${ }^{\mathrm{xxx}}$

Philon reprend ici les acquis de la section XI de l'Enquête sur l'entendement humain : le degré d'évidence de l'inférence d'une cause à partir de son effet, est proportionné au degré de ressemblance que soutient cet effet avec l'autre effet dont on a expérimenté la conjonction avec une cause. Il retrouve ainsi le problème inhérent à l'argument du dessein présenté dans cette section : la disproportion entre l'effet et la cause inférée. En effet, quand nous inférons une cause particulière d'un effet, il nous faut proportionner l'un à l'autre, et l'on ne peut nous accorder d'attribuer à la cause que les qualités qui suffisent exactement à produire l'effet ${ }^{\mathrm{xxxi}}$.

Or, le monde ne se présente pas comme un produit parfait et harmonieux : l'ordre y connaît de nombreux écarts, la vie humaine connaît bien des maux. De l'observation de ce monde imparfait à l'inférence de l'intention d'un être infiniment parfait, il y a un saut : on attribue à la cause bien plus de qualités que l'effet n'en manifeste. Dans la onzième partie des 
Dialogues, Philon examine cette disproportion entre le monde considéré comme un effet et le dessein divin pour invalider la pertinence de l'inférence.

Cette disproportion évidente entre cause et effet dans le théisme expérimental intervient-elle dans le contractualisme ? On pourrait objecter, à première vue, que le saut qui va de la société au contrat paraît moindre que celui qui remonte du monde à Dieu. Après tout, il ne s'agit que d'expliquer un phénomène humain, la société, par une association volontaire passée entre des hommes. Il ne semble pas ici que la cause supposée contienne plus de qualités que n'en présente son effet. Or, c'est précisément un tel écart injustifiable qui est mis en évidence dans l'essai «Du contrat originel», lorsque Hume qualifie le contractualisme de système philosophique et raffiné, fruit d'esprits chimériques, que rien ne vient justifier dans le vaste monde $^{\text {xxxii }}$. Et en effet, lorsque l'on observe les différents systèmes politiques en place, que voit-on? Des changements continuels, des expansions et des dissolutions, des émigrations et des alliances de dynasties, des révolutions et des conspirations. Bref, l'observation des sociétés politiques comme lieux du changement brutal et de la violence, interdit, en raison de la règle de proportion, d'inférer le contrat comme cause. Le contrat contient effectivement plus de qualités que n'en manifeste son effet supposé : la stabilité et le consensus, l'harmonie et l'approbation. C'est dans cette perspective que Hume mobilise l'histoire et l'expérience pour insister sur le caractère incertain, ambigu et arbitraire des institutions qui interdit l'inférence d'un quelconque contrat. Le contrat est ici débouté comme origine causale historique.

Ainsi le rejet du contractualisme s'enracine-t-il dans l'analyse empirico-sceptique de la causalité : une cause, quelle qu'elle soit, ne peut jamais être établie a priori, mais seulement a posteriori par observation directe ou par inférence adoptant la règle de proportion. Dans la mesure où le contrat n'est pas observable et où l'ordre social qu'il prétend 
fonder contient bien moins de qualités qu'il ne lui en procurerait s'il en était l'origine, le contractualisme n'est pas une hypothèse épistémologiquement valide.

La quatrième partie des Dialogues sur la religion naturelle constitue la réfutation la plus redoutable de l'argument du dessein en faisant porter la critique explicitement sur la notion d'intention. Quand bien même, on admettrait la pertinence de l'explication par les causes finales, et bien on n'expliquerait rien du tout ! Le problème de l'argument du dessein ne réside pas tant dans l'analogie douteuse et la disproportion sur laquelle il repose, que dans sa faiblesse explicative. Les commentateurs ${ }^{\mathrm{xx} x i i}$ analysent habituellement ce second argumentaire de Philon comme conséquence logique du changement de tactique opéré par Cléanthe qui, après avoir exposé l'argument du dessein comme une démonstration rigoureuse et s'être fait débouté par le rappel de la règle de proportion à laquelle doit se soumettre toute inférence pour être valide, en vient à le présenter comme une croyance instinctive. Si ce commentaire est pertinent, il importe néanmoins de souligner que Philon, en reconnaissant avec Cléanthe qu'il n'est pas si facile de ne pas comparer le monde à un produit ordonné, va ruiner définitivement l'explication par les causes finales. Il n'admet l'analogie que pour mieux la retourner contre elle-même.

Pour mieux comprendre la portée de cette critique de la notion d'intention, il convient de se rappeler les analyses humiennes consacrées à la raison. Dans le Traité de la nature humaine, il la présente comme une sorte de cause ${ }^{\mathrm{xxxiv}}$, de sorte que du moment où il y a conjonction constante entre motivations et actions, on peut aisément parler de causalité au même titre que lorsque l'on établit des relations causales entre les corps ${ }^{\mathrm{xxxv}}$. La dimension intentionnelle de la causalité du dessein est appréhendée dans son effectivité productrice. Cette identification entre la causalité morale et la causalité physique montre que la première 
n'est absolument pas distincte de la seconde : non seulement l'intentionnalité ne jouit d'aucun privilège par rapport à la causalité efficiente, mais elle est une de ses modalités.

Les Dialogues sur la religion naturelle tirent les conséquences de cette subsomption : si l'intentionnalité est une cause comme une autre, elle ne saurait être une cause première. La quatrième partie des Dialogues est en ce sens décisive : elle promeut un type d'explication alternatif dissociant ordre et finalité et discréditant la notion d'intention. En effet, ce n'est pas seulement le monde que l'on ne peut pas expliquer de manière satisfaisante par les causes finales, mais également les produits de l'art humain. Si ces derniers sont causés par des idées, la représentation qu'en ont les personnes qui les créent, une question demeure en suspens, marquant l'insuffisance du finalisme et déboutant ses prétentions théoriques : comment les idées se mettent-elles en place dans le cerveau de l'artisan ? Comment est produite l'intention? Que l'intention soit une cause, pourquoi pas ? Mais elle-même, par quoi est-elle causée ? L'explication par le recours à une volonté intentionnelle est une explication partielle et provisoire, qui ne fait que réitérer la question initiale.

Ainsi le retors Philon ne feint-il l'accord avec son adversaire que pour mieux l'acculer. Admettons que l'analogie de Cléanthe entre le monde et un produit manufacturé soit valide sous prétexte que dans les deux cas, il y a ordonnancement et qu'il faille expliquer l'ordre par une intention extérieure. Dans ce cas-là, l'analogie doit être étendue au monde mental : qui ordonne nos idées dans la tête ? Le dessein lui-même demande à être expliqué.

Si la raison (j'entends la raison abstraite tirée de recherches a priori) n'est pas pareillement muette sur toutes les questions concernant la cause et l'effet, au moins se risquera-t-elle à rendre ce jugement qu'un monde mental ou univers d'idées requiert une cause tout autant que le fait un monde matériel ou univers d'objets et, s'il est semblable dans son arrangement, qu'il requiert nécessairement une cause semblable. Car qu'y a-t-il en l'affaire qui donnerait lieu à une conclusion ou à une inférence différente ? À un point 
de vue abstrait, ces deux mondes sont entièrement semblables; et nulle difficulté ne s'attache à l'une des suppositions, qui ne soit pas commune aux deux ${ }^{\mathrm{xxxvi}}$.

À ce point du dialogue semble poindre le danger d'une régression à l'infini dans la recherche des causes quand l'intention est en jeu, qu'il s'agisse d'intention divine ou d'intention humaine.

Avant de présenter la promotion d'un autre type d'explication proposée par Philon et étendue par analogie du monde mental au monde matériel, il convient de voir comment la dénonciation de ce questionnement sans fin anime la seconde partie de l'essai «Du contrat originel ». En effet, Hume montre que le respect des promesses ne peut pas constituer le fondement du devoir d'allégeance, dans la mesure où, lui aussi, demande à être expliqué.

Nous sommes tenus d'obéir à notre souverain, dit-on, parce que nous lui avons donné tacitement notre parole là-dessus. Mais pourquoi sommes-nous tenus à tenir parole ? (...) L'obligation d'allégeance ayant même force et même autorité que l'obligation de fidélité, nous ne gagnons rien à ramener l'une à l'autre $e^{\mathrm{xxxvii}}$.

Ainsi le contrat se voit-il débouté dans sa prétention normative dans la mesure où l'on est toujours en droit de lui demander : pourquoi obligerait-il ? La norme ne peut se suffire à elle-même en se présentant comme déjà constituée. Le contractualisme commet un diallèle.

Comment Philon propose-t-il d'échapper à cette régression à l'infini ? La seule manière de ne pas chercher une cause à l'ordonnancement de nos idées aboutissant à une représentation causale, est de dire que nos idées s'ordonnent d'elles-mêmes. Qu'il y a un principe d'ordre immanent. Et en effet, que révèle la considération des produits de l'art humain, sinon que l'intention productrice émerge peu à peu suite à de multiples essais infructueux, à l'imitation, ou encore à un progrès lent et continu sous la pression des circonstances ?xxviii Bref, l'intention est moins une cause que l'effet émergent d'une histoire. 
Mais si Cléanthe pouvait dresser une analogie entre monde et machine sous prétexte qu'il s'agissait de deux formes d'ordre, il faut le faire également entre le monde mental et le monde matériel, de sorte que l'on peut inférer la cause de l'un à partir de la cause de l'autre ! Philon, faisant l'économie de tout recours à l'intentionnalité, peut alors présenter l'hypothèse épicurienne d'une causalité immanente à chaque phénomène ordonné comme l'hypothèse la plus plausible.

Et si j'étais dans l'obligation de défendre un système particulier de cette nature (ce que je ne ferais jamais volontiers) je n'en juge aucun qui soit plus plausible que celui qui assigne au monde un principe d'ordre éternel et inhérent, bien qu'accompagné de grandes et continuelles révolutions et altérations. Il résout immédiatement toutes les difficultés; et si la solution, du fait de sa généralité n'est pas entièrement complète et satisfaisante, c'est du moins une théorie à laquelle nous devons, tôt ou tard, avoir recours quelque système que nous embrassions. Comment les choses auraient-elles pu être ce qu'elles sont, s'il n'y avait pas un principe d'ordre, originel et inhérent, résidant quelque part dans la pensée ou la matière? Et il est parfaitement indifférent de savoir à laquelle des deux nous donnons la préférence $e^{\mathrm{xxxix}}$.

Il s'agit là d'une véritable précepte méthodologique : pour expliquer un ordre quelconque, l'ordre des idées, l'ordre du monde ou l'ordre social, il faut l'expliquer par un principe d'ordre inhérent au phénomène, de même niveau que lui, sans chercher une cause ultime ou une raison extérieure, faute de quoi l'on est menacé d'une stérile régression à l'infini. Ainsi Hume subvertit-il du tout au tout la notion de principe : lorsqu'il parle de principe, il n'entend jamais un principe rationnel, c'est-à-dire un fondement, mais seulement un principe factuel dont il faut décrire le déploiement immanent au phénomène qu'il explique, sans que l'on puisse en fournir la raison dernière. L'organisation se pense indépendamment du finalisme ${ }^{\mathrm{xl}}$. La causalité explicative de l'ordre est simplement efficiente. Et encore ne faut- 
il pas trop accorder à cet ordre : il est plus ordonnancement qu'ordre, processus que résultat définitif.

L'argument du dessein apparaît comme doublement insuffisant : d'une part, le dessein ne respecte pas comme mode particulier de cause empirique la règle de proportion, et d'autre part, quand bien même il serait inféré de manière valide en respectant cette règle, il n'explique rien. Or, si l'intention demande à être expliquée, elle ne jouit d'aucun privilège pour rendre compte des phénomènes ordonnés. Puisque la seule manière d'échapper à une recherche de causes à l'infini est de poser un principe d'organisation interne, il faut le faire pour tous les phénomènes ordonnés. Chaque ordre doit être expliqué de manière immanente. Dans la mesure où le recours à l'hypothèse égoïste fait du contractualisme la transposition politique de cet argument du dessein, la critique humienne du contractualisme est motivée avant tout par sa faible portée épistémologique. Il existe une disproportion manifeste entre les sociétés et leur prétendue origine. Et si l'on admettait une telle origine, l'interrogation ne serait pas close pour autant : en effet, l'on devrait toujours se demander comment la promesse peut émerger et constituer une obligation. Si la règle de proportion met à mal la notion de contrat comme événement historique, les difficultés de la notion d'intention interdisent de le penser comme origine normative de l'obligation politique. Celle-ci ne peut donc jamais être pensée de manière a priori mais toujours au sein d'un processus. Pour échapper à ces difficultés, Hume propose donc en lieu et place du contractualisme une hypothèse génétique. La question est alors de savoir quel est le principe d'ordre immanent au domaine politique.

\section{Une histoire naturelle du politique}


Hume, selon la distinction que fixera L'histoire naturelle de la religion ${ }^{\text {xli }}$, explique dans le livre 3 du Traité de la nature humaine le phénomène politique par le recours à l'origine et non au fondement. Par là, il faut entendre qu'il rend compte, et non pas raison, du phénomène par un principe d'ordre immanent et factuel. Dans la mesure où la société politique est un phénomène humain, il s'agira d'un principe de la nature humaine, la sympathie. Comme le souligne Jennifer Herdt, tout l'effort du Traité est de promouvoir un concept non-théologique qui peut rendre compte d'un tel phénomène sans l'intervention d'une quelconque finalitéxlii. L'origine n'étant pas un événement normatif mais le déploiement d'un principe dans le temps, on peut alors parler d'histoire naturelle. Ainsi Hume décrit-il une histoire où la sympathie se modifie peu à peu et produit un effet processuel, en réajustement permanent, la société.

De manière remarquable, le point de départ de cette histoire du politique n'est pas l'individu isolé de l'état de nature, qui serait en position extérieure par rapport à la société, mais l'individu vivant dans une famille. A la place du triptyque égoïsme-raison-volonté du contractualisme, Hume fait jouer une autre séquence où la sympathie partiale se modifie en sympathie élargie: s'il ne nie pas l'intervention de la raison et de la volonté dans cette instauration du politique, il le fait en les considérant comme causes secondaires et immanentes au processus, éduquées par la conjugaison de la sympathie et de l'habitude.

- l'évidence du sens de l'intérêt commun dans la famille.

La famille joue un rôle décisif dans cette histoire naturelle du politique : c'est dans cette première forme de société, stabilisée par la sympathie, que les hommes peuvent prendre conscience des avantages la vie en commun. Si les hommes n'avaient pas déjà un penchant à la vie sociale, et s'ils n'avaient pas éprouvé comme impressions le plaisir et les avantages à vivre ensemble, ils n'auraient jamais pu en avoir l'idée. L'argument empirique de 
base selon lequel on ne peut avoir l'idée d'une chose si l'on n'en a pas d'abord éprouvé l'impression, argument présenté par Livingston comme le premier principe de la philosophie de Hume ${ }^{\text {xliii }}$, s'avère d'une importance décisive : jamais un individu isolé ne pourrait avoir idée des avantages de la vie en société. L'hypothèse égoïste liée au contractualisme méconnaît une donnée anthropologique capitale : l'homme vit naturellement en société, fût-ce sous la forme fruste qu'est la famille. Hume, sans aucune ambiguïté, considère cette dernière comme naturelle.

L'homme étant né dans une famille, est contraint de rester en société, par nécessité, par inclination naturelle et par habitude $e^{\text {xliv }}$.

Les hommes naissent toujours dans une société, familiale au moins, et sont formés par leurs parents à certaines règles de conduite et de comportement ${ }^{\mathrm{xlv}}$.

La première union entre hommes et femmes assurée par l'instinct sexuel est renforcée par le deuxième type d'union qu'elle entraîne, celui entre parents et enfants. Or la sympathie est intimement liée à l'amour des parents pour leurs enfants. Mieux, ce dernier ne peut se comprendre sans elle. Alors que l'amour se caractérise de manière générale comme une passion indirecte reposant sur une double relation d'impressions et d'idées (la qualité agréable d'une personne autre que moi produit une impression agréable et l'idée de cette personne), on observe que l'on peut aimer une personne dépourvue qualité agréable. Ceci est particulièrement vrai dans le cas de l'amour des parents vis-à-vis de leur progéniture : les parents aiment leurs enfant quand bien même ils n'ont rien d'aimable. En quoi le lien du sang, est-il propre à susciter un tel amour ? La réponse à cette question est cruciale en donnant à comprendre comment se stabilise le lien social dans le prolongement de la première relation biologique entre les individus.

L'explication de Hume fait intervenir la sympathie et l'incapacité de la subjectivité à se soutenir elle-même. La sympathie, comme l'a établi le livre 2 du Traité de la 
nature humaine, permet d'éprouver comme impression ce qu'éprouve autrui. Or la vivacité de l'impression est source de plaisir car elle donne de l'agitation à notre esprit.

Une sympathie envers les autres n'est agréable que par le branle qu'elle donne aux esprits, puisqu'une sympathie aisée et les émotions correspondantes sont seules communes à la parenté, à la familiarité, et à la ressemblance ${ }^{\mathrm{xlvi}}$.

Il se trouve que l'on sympathise d'autant plus avec quelqu'un qu'il nous est relié par parenté, familiarité ou ressemblance. En effet, la ressemblance et l'accoutumance à une personne rendent plus aisée l'inférence de ses émotions à partir de son comportement. Puisque les liens de sang englobent ces deux relations, (de manière générale, on ressemble à ces enfants et l'on est habitué à vivre avec eux), l'on sympathise très facilement avec les gens de sa famille: l'on éprouve aisément comme impression ce qu'ils éprouvent eux-mêmes comme impression. Cette facilité est source de plaisir dans la mesure où elle fournit à l'esprit qui n'est jamais à lui-même son propre fonds, la vivacité qui lui permet de prendre conscience de lui-même. Elle peut donc produire l'amour sans que la personne aimée ait une qualité agréable. La sympathie assure ainsi un premier lien social au sein de la famille.

C'est dans cette étude de la famille comme embryon de société qu'apparaît la notion d'intérêt. Entre les parents s'établit un nouveau lien, le souci qu'ils ont de leur progéniture, qui se rajoute à l'instinct sexuel.

Ce nouvel intérêt devient aussi un principe d'union entre parents et enfants et il donne forme à une société plus nombreuse, dans laquelle les parents gouvernent à cause de l'avantage que leur donne leur force et leur sagesse supérieures, et dans laquelle, en même temps l'affection naturelle qu'ils ont pour leurs enfants retient l'exercice de leur autorité $^{\mathrm{xlvii}}$. 
En vivant en famille, les hommes prennent ainsi conscience des avantages de la vie en société. Dans la mesure où tout homme a été enfant avant d'être adulte, et est toujours né dans une famille, il faut dire que la prise de conscience de ses avantages passe par l'habitude de l'obéissance. Mais cette prise de conscience n'est rendue possible que parce que les membres d'une même famille, du fait de leur ressemblance et de leur familiarité, partagent le même intérêt. En vertu de la forte sympathie des époux entre eux, des enfants entre eux et des parents avec les enfants et vice-versa, l'intérêt commun est évident. Ce qui fait plaisir aux autres membres fait plaisir à chacun et vice-versa. La sympathie est donc à l'origine de la prise de conscience de l'avantage qu'il y a à vivre en société : il est très facile de trouver un accord en raison de la très grande facilité à se mettre à la place les uns des autres. C'est pourquoi on trouve des rudiments de justice dans la famille.

Il est capital de noter que Hume ne dit pas autre chose sinon que la première convention a pour lieu la famille. En effet, la convention peut se définir comme « un sens de l'intérêt que l'on suppose commun à tous » ${ }^{\text {xlviii }}$, et c'est par ce sens de l'intérêt que peuvent être adoptées les règles de justice. Mais encore faut-il que le lien entre règles de justice et intérêt commun soit évident, or seule la famille permet d'établir ce lien. Par sympathie, chaque membre de la famille a conscience de l'intérêt des autres et se l'approprie. Donc contrairement à la perspective contractualiste, il n'y a pas besoin de l'intervention de la raison pour expliquer ce qui est de l'ordre d'une convention spontanée et relativement immédiate. L'intérêt commun n'est pas choisi rationnellement mais est vécu naturellement du fait de la sympathie.

Dans la famille, intérêt commun et intérêt personnel se recouvrent de telle sorte que l'invention du premier se fait spontanément. Si les avantages de cette coopération n'avaient pas été éprouvés au sein de la famille, jamais les individus n'auraient pu avoir l'idée 
de mettre en place des conventions plus élaborées de justice. Le rôle de la famille, décisif dans la genèse de la société, permet de comprendre comment la première action coopérative du point de vue généalogique est établie de manière spontanée, naturelle et immanente grâce au principe de la nature humaine qu'est la sympathie.

- La(es) convention(s) entre familles n'éprouvant pas de sympathie entre elles.

Dans cette étape, Hume fait intervenir la notion substitutive à celle de contrat, la notion de convention. La sympathie se corrige d'elle-même pour inclure la famille au sein d'un ordre supérieur, la société civile régie par les règles de justice, et éventuellement cette dernière au sein d'une société politisée.

Que l'homme soit toujours membre d'une famille et qu'il préfère ses proches aux membres plus éloignés est un facteur de déstabilisation. En effet, dans une telle configuration, il y a risque potentiel de guerre entre les différentes familles en présence. Le nombre de biens extérieurs étant limité, chaque famille cherche à s'en emparer, ce qui entraîne une guerre non de tous contre tous selon la formule de Hobbes, mais de famille contre famille, nuisant aux intérêts de chacune. Les membres de chacune risquent d'être égorgés et les biens pillés.

La guerre, comme conséquence du déploiement de la sympathie, en appelle à un remède. Les hommes décident de se mettre d'accord entre eux pour garantir ces biens extérieurs.

Cela ne peut être fait d'aucune autre manière que par une convention souscrite par tous les membres de la communauté afin de conférer de la stabilité à la possession de ses biens extérieurs et de laisser chacun dans la paisible jouissance de ce qu'il a pu acquérir par sa bonne fortune ou son labeur ${ }^{\mathrm{xlix}}$. 
Hume insiste sur le fait que la convention comme accord entre les hommes ne nécessitant pas de promesses mais un ajustement progressif des comportements les uns aux autres, requiert intelligence et réflexion. Il est de l'intérêt de chacun de respecter la propriété pour mettre fin à la guerre entre les clans, dommageable à chacun.

Le remède, dès lors, ne provient pas de la nature mais de l'artifice ou, pour parler plus exactement, la nature fournit, dans le jugement et l'entendement, un remède à ce qui est irrégulier et malencontreux dans ces affections ${ }^{1}$.

Mais de manière remarquable, le fait que ce soit la nature qui fournisse le remède aux affections indique qu'il n'y a pas de rupture introduite par une cause extérieure au processus : l'intelligence et la réflexion interviennent comme causes secondaires immanentes à l'ordonnancement réalisé par la sympathie. La sympathie, principe d'ordre devenu momentanément facteur de désordre, se corrige d'elle-même en fournissant la solution et la garantie de son applicabilité.

Un élément très important de la convention est en effet, le fait qu'elle s'inscrive dans la longue durée ${ }^{\text {li }}$. Elle n'est pas le fruit d'une décision immédiate mais le résultat d'un ajustement qui a pris du temps. L'homme comprend peu à peu qu'il est de son intérêt de contraindre la passion de l'intérêt personnel pour mieux la satisfaire, qu'il doit renoncer à satisfaire de manière immédiate sa famille pour mieux pouvoir la satisfaire sur le long terme. La passion en appelle à la réflexion qui la régule pour son bien. Ainsi la réflexion est-elle en position de médiation. Mais il ne faut pas oublier l'analyse menée précédemment par Hume sur la famille. L'intérêt ajoute une précision qui permet de comprendre que la réflexion se déploie sur fond de sympathie. En effet, si la règle de la stabilité des possessions peut être adoptée par convention, c'est dans la mesure où elle est évidente. Ceci ne signifie cependant pas que ce soit l'entendement qui le comprenne. Non, si l'invention de cette règle est 
évidente, c'est dans la mesure où elle a été expérimentée comme telle dans la famille où elle se met en place spontanément du fait de la sympathie immédiate entre ses membres. Chacun se dispense de s'emparer du bien de l'autre puisqu'il n'y a pas de relation de rivalité entre les membres : tout ce qui procure du plaisir à mes proches m'en procure aussi. Il est donc totalement inutile de s'emparer du bien de ses proches pour avoir du plaisir. Lorsque Hume écrit que «les hommes, par leur première éducation en société », deviennent « conscients des avantages infinis qu'elle procure $»^{\text {lii }}$, il faut entendre que la sympathie conjuguée à l'habitude est formatrice pour l'intelligence. C'est parce que les hommes ont éprouvé par elle le plaisir qu'il y a à vivre en société, fût-ce dans une société aussi rudimentaire que celle de la famille, qu'ils peuvent décider d'élargir le groupe dans lequel ils vivent. Hume indique à deux reprises dans le Traité de la nature humaine que les hommes décident d'agrandir la sphère sociale dans laquelle ils vivent non seulement pour protéger leur famille mais parce qu'ils éprouvent du plaisir à vivre en sociétéliii

Mais l'anticipation rationnelle des avantages et du plaisir que l'on peut tirer avec ses proches de la mise en place artificielle de la convention qui régnait spontanément dans la famille, implique elle aussi la sympathie. La convention est applicable dans la mesure où tous les hommes se ressemblent, où la nature humaine est une. L'homme pense en effet qu'il satisfera mieux son intérêt et celui de sa famille en souscrivant une convention car il suppose que les autres membres raisonnent de la même manière. Il suppose que, s'il s'abstient de s'emparer de leurs biens, ils feront de même, et ce, pour les mêmes raisons que lui. Cette convention n'est pas de la nature d'une promesse, car les promesses elles-mêmes naissent des conventions humaines. Ce n'est qu'un sens général de l'intérêt commun, sens que les membres de la société expriment les uns aux autres, et qui les conduit à régler leur comportement selon certaines règles. 
J'observe qu'il sera de mon intérêt de laisser autrui en possession de ses biens, pourvu qu'il agisse de même avec moi. Il a conscience d'avoir le même intérêt à réguler sa conduite. Quand ce sens commun de l'intérêt est mutuellement formulé et qu'il est connu des deux, il produit une résolution et un comportement qui lui correspondent. Et cela, on peut l'appeler avec assez d'à propos une convention ou un accord entre nous, bien que fasse défaut la médiation d'une promesse, puisque les actions de chacun d'entre nous font référence à celle de l'autre et sont accomplies en supposant que de l'autre côté, on doit aussi accomplir quelque chose $e^{\text {liv }}$.

Le ressort de la convention est la ressemblance entre les hommes. Les parties qui nouent entre elles une convention sont conscientes qu'elles ont le même intérêt à la respecter. Et ce, dans la mesure où la partialité est la qualité commune à tous les hommes. Je fais confiance à l'autre car l'autre n'est jamais un étranger radical pour moi ${ }^{\mathrm{lv}}$. Ce fond de ressemblance première, le fait que tous les hommes éprouvent de la sympathie pour leurs proches, permet la communication, c'est grâce à lui que l'on peut «mutuellement formuler » ce sens de l'intérêt commun et se comprendre.

La célèbre illustration de ce qu'est une convention par l'exemple des rameurs est significative : les rameurs peuvent ajuster leur mouvement l'un à l'autre, dans la mesure où ils veulent aller spontanément dans la même direction. Donc parce qu'il y a une ressemblance initiale. Si l'un ne voulait pas bouger alors que l'autre voulait se déplacer, et si chacun voulait aller dans une direction opposée, l'ajustement progressif des conduites serait tout simplement impossible. Si les hommes peuvent se comprendre entre eux, c'est qu'il existe comme le dit Annette Baier «une coopération en quelque sorte instinctive et une communication automatique $\gg^{\text {lvi }}$. Ce qui nous permet de comprendre que nous partageons le même intérêt et que nous pouvons nous faire confiance, est l'expression similaire de nos sentiments parallèles. 
La convention institue donc une société civile en formulant des règles de justice concernant la propriété. Cependant si deux rameurs peuvent s'entendre aisément pour pagayer de concert, la chose paraît autrement plus difficile pour un équipage plus nombreux ${ }^{\text {lvii }}$. Pour pallier ce qu'ils ne peuvent guérir directement, les hommes peuvent alors instituer un gouvernement. De la sorte, ils sont contraints de respecter les biens des autres et évitent ainsi une guerre qui leur serait dommageable. Il s'agit là d'une institutionnalisation d'ordre supérieur qui renforce la convention initiale, dans la mesure où l'habitude de vivre dans une société politique accroît le sentiment des avantages qu'il y a à respecter les règles de justice et conduit à reconnaître cette société comme légale et obligatoire. L'habitude consolide donc la sympathie ${ }^{\text {lviii }}$ en faisant inférer une relation de nécessité entre le gouvernement et l'obéissance. La répétition de la conjonction devient connexion nécessaire. Cette institutionnalisation est moins le fruit d'une décision que le fruit des circonstances : si les règles de justice sont sans cesse bafouées, c'est naturellement que l'on se tourne vers une tierce partie pour les faire respecter.

Aucun contrat ou accord, c'est évident ne fut expressément conclu en vue de la soumission générale - c'est là une idée qui passe de fort loin l'entendement des sauvages : chaque exercice de l'autorité de la part du chef doit avoir été particulier et appelé par les exigences de chaque cas ; l'utilité sensible qui résultait de son entremise rendit cet exercice de jour en jour plus fréquent, et cette fréquence produisit par degrés un assentiment habituel dans le peuple ${ }^{\mathrm{lix}}$.

La sympathie intervient dans cette seconde étape comme éducatrice de l'intelligence, c'est-à-dire comme moteur de l'invention progressive des institutions. Grâce à elle, les hommes expérimentent les avantages de la vie en société, d'abord dans la famille, puis dans la société civile, et enfin dans la société politique. La convention, à la différence du contrat, n'est pas l'anticipation volontaire et rationnelle circonscrite dans le temps d'un 
moyen pour satisfaire une certaine fin, mais le déploiement spontané et progressif d'un principe de la nature humaine, la sympathie, se corrigeant grâce à l'habitude sous la pression des circonstances, pour mieux se satisfaire. Ses inventions, règles de justice et gouvernement, sont le produit de l'inventivité de ce principe qui se régule de lui-même.

\section{- la convention comme obligation morale}

La troisième étape de la formation de la société consiste dans la moralisation de la convention et dans l'invention de normes proprement sociales. La sympathie se dépasse à nouveau elle-même : la partialité brutale est obligée de devenir partialité contenue pour mieux se satisfaire. En se contenant, elle devient sympathie élargie. Si le premier motif de l'association est la poursuite de son intérêt et de celui de ses proches, ce motif est susceptible de devenir sens de l'intérêt général. Cette transformation rend compte du fait que l'on associe la notion de vertu à celle de justice et celle de vice à l'injustice. La sympathie apparaît ainsi comme la source des distinctions morales ${ }^{\mathrm{lx}}$.

Ainsi, l'intérêt personnel est le motif originel de l'institution de la justice; mais une sympathie avec l'intérêt public est la source de l'approbation morale qui accompagne cette vertu $^{\text {lxi }}$.

L'homme finit par éprouver du plaisir à ce qui est bénéfique à la société en tant que telle, indépendamment de son propre intérêt. Hume n'hésite pas à qualifier ce sens moral de naturel dans la mesure où il se produit infailliblement même s'il n'est pas immédiat.

D'après ces principes, nous pouvons facilement écarter toute contradiction qui peut sembler exister entre la sympathie étendue, dont nos sentiments de la vertu dépendent, et cette générosité limitée qui est naturelle aux hommes, comme je l'ai souvent fait remarquer, et que supposent la justice et la propriété, conformément au raisonnement qui a précédétii. 
Quelles sont les modalités qui permettent d'éprouver du plaisir non seulement à ce qui fait plaisir à ses proches mais également à ce qui est bénéfique à la société en général ? La fréquentation des autres conduit à élargir son point de vue. Les hommes peuvent communiquer de manière immédiate du fait qu'ils partagent quelque chose en commun, la partialité. Cette composante universelle de la nature humaine autorise les hommes à se faire confiance et facilite la communication entre eux. Mais à partir du moment où l'on n'est plus cantonné à la sphère des proches et où l'on parle avec des gens pour lesquels l'on n'a pas de sympathie de prime abord, l'on constate qu'

$$
\begin{aligned}
& \text { (...) il est impossible que nous puissions jamais converser ensemble en des termes } \\
& \text { raisonnables, si chacun de nous considère les personnes et les caractères uniquement tels } \\
& \text { qu'ils apparaissent de son point de vue singulier. Par conséquent, afin de prévenir ces } \\
& \text { contradictions continuelles et de parvenir à un jugement des choses qui soit plus stable, } \\
& \text { nous choisissons des points de vue ferme et généraux et, dans nos pensées, nous nous y } \\
& \text { plaçons toujours, quelque puisse être notre situation extérieure }{ }^{\text {lxiii }} \text {. }
\end{aligned}
$$

Ainsi la partialité se corrige-t-elle d'elle-même : c'est elle qui fonde la possibilité de la confiance et les conditions de sa propre extension. Elle se réfléchit en se stabilisant, c'est-à-dire en se généralisant. Ceci ne signifie pas, bien évidemment, que l'on est prêt à sacrifier son intérêt pour les autres, mais il n'empêche que le point de vue des autres s'insinuant peu à peu en nous, nous fait former une certaine norme générale. L'habitude de la partialité des autres produit l'impartialité. Celle-ci n'est pas première mais construite.

Le seul point de vue en lequel nos sentiments coïncident avec ceux des autres, c'est lorsque nous considérons la tendance d'une passion à être avantageuse ou dommageable pour ceux qui ont un rapport direct ou une relation immédiate avec ceux qui la possède. Et bien que cet avantage ou ce dommage soient souvent très éloignés de nous, parfois, ils 
sont pourtant très proches et nous intéressent fortement par sympathie. Cet intérêt, nous

l'étendons sans difficultés aux autres cas semblables, et quand ils sont très éloignés, notre sympathie est proportionnellement plus faible, notre éloge ou notre blâme sont plus vagues et plus hésitants ${ }^{\mathrm{lxiv}}$.

De même que notre jugement à force d'expériences répétées produit de la stabilité à partir de la variété des perceptions (un objet dont la taille change selon que nous nous rapprochons ou nous éloignons doit être considéré en fait comme le même objet), il produit de la stabilité à partir de la variété des émotions. En côtoyant les autres, on forme peu à peu des notions générales qui sont les normes sociales : ce que l'on doit approuver en société est ce qui est bénéfique à la société, ce que l'on doit désapprouver est ce qui est nuisible. De telles normes sociales forgées empiriquement orientent notre conduite par leur caractère d'obligation. Le point de vue général intégré par chacun au fur et à mesure de sa formation devient un principe d'action.

Ces trois étapes de la vie sociale mettent en lumière de manière manifeste le rôle de la sympathie comme principe d'ordre inhérent au politique. L'explication permise par ce principe de la nature humaine est entièrement immanente au processus décrit : entre la cause et l'effet, il n'y a aucune rupture, contrairement à celle introduite par une explication de type finaliste. La sympathie comme facteur de relation entre époux et comme facteur de relation entre parents et enfants assure un premier lien social où intérêt personnel et intérêt commun se recouvrent strictement. Cette relation première permet à chacun d'éprouver les bienfaits de la vie en société : sans cette sociabilité première, l'homme n'aurait jamais pu prendre la décision de vivre dans une société plus vaste. La sympathie comme lien social élémentaire est donc le motif de la convention entre les familles, mais cette convention, elle-même, n'est possible que parce que les hommes peuvent se mettre d'accord entre eux et se faire confiance. Or s'ils 
peuvent se faire confiance, c'est parce qu'ils ont quelque chose en commun : l'intérêt pris à eux-mêmes et à leurs proches. Chaque membre des différentes familles peut rationnellement attendre que l'autre respecte l'accord. Réflexion et anticipation sont les produits de la sympathie. La convention rationnellement choisie institue, sur la base du lien social, un espace commun. Cet espace commun engendre une correction de la sympathie en lui permettant de s'étendre : la partialité de chacun est obligée de se réfléchir pour coïncider avec celle des autres. Un schème est ainsi instauré, la société comme puissance morale, source d'obligations. La société est un phénomène ordonné de manière immanente.

Ainsi espérons-nous avoir montré la pertinence d'éclairer la philosophie politique de Hume par ses analyses épistémologiques. Les Dialogues sur la religion naturelle permettent de dégager les thèses méthodologiques liées à son analyse de la causalité qui sont impliquées dans sa philosophie politique. Le rejet du contractualisme doit se comprendre comme la critique d'une explication par les causes finales et non comme une position idéologique. La finalité est introduite par une considération anthropologiquement fausse de la psychologie humaine. En faisant de l'intention raisonnable de l'individu solitaire et égoïste la cause transcendante du processus, le contractualisme introduit une double hétérogénéité : entre la cause et l'effet, et entre le moyen et la fin. La société comme effet de la volonté raisonnable des individus (la cause) est le moyen pour satisfaire l'amour de soi (la fin). Mais ce faisant, les contractualistes ne font que reproduire la difficulté des théistes : comment cette intention a-t-elle pu émerger ? Comment le sens du respect de l'engagement s'est-il constitué ? Si les contractualistes répondent que les différentes idées qui composent la raison des individus se mettent en ordre d'elles-même, alors cette réponse signifie que l'on peut expliquer l'ordre dans ses différentes manifestations de manière immanente, sans avoir 
recours à une cause extérieure visant une fin pour laquelle l'effet causé n'est qu'un moyen. Sans cela, l'explication restera toujours inachevée et insatisfaisante. Que les contractualistes n'aient pas voulu expliquer les sociétés politiques actuelles par le recours au fondement du contrat social, mais seulement une société politique idéale, ne permet pas d'invalider la critique humienne. Ce que rejette précisément Hume est le contrat social comme fondement réel ou possible. Au principe de raison, Hume substitue un principe de la nature humaine, la sympathie, dont le cours naturel et ordinaire configure et reconfigure de manière immanente la société comme famille, société civile, société politique et société morale. Chaque obligation est le fruit d'une production causale, le résultat d'une histoire.

Deux leçons se dégagent de cette critique :

- Une leçon méthodologique générale, aussi modeste qu'exigeante, qui anime l'œuvre entière de Hume. L'explication de l'ordre, de tous les ordres, doit se cantonner au comment en renonçant au pourquoi. Il ne faut pas chercher de principe explicatif transcendant, c'est-à-dire de cause finale, mais un principe explicatif immanent, c'està-dire une causalité efficiente. C'est en limitant ses prétentions que l'explication peut parvenir à un résultat.

- Une leçon sociologique particulière découlant de l'application de cette méthode. Le social doit être expliqué par le social. Le principe d'ordonnancement immanent, seul principe explicatif pertinent, appartient en effet au même niveau de réalité que le phénomène ordonné. Si l'individu veut la société, ce n'est pas pour satisfaire son égoïsme, mais uniquement par l'action en lui du principe de mise en relation qu'est la sympathie. L'analyse de Hume retentit d'un écho inattendu dans l'œuvre de Durkheim $^{\mathrm{lxv}}$ : la société à travers les pratiques individuelles se veut elle-même, fût-ce sous un mode tâtonnant. 
${ }^{i}$ Cf. « Of the Original Contract», in The Philosophical Works of David Hume, ed. by T.H. Green and T. H. Grose (GG), London, 1874-1875, reprint Darmstadt, Scientia Verlag Aalen, 1992, vol. IV, note 1, p. 460, trad. de F. Grandjean (FG) in Discours politiques, Mauvezin, Trans-Europ-Repress, 1993, note 422, p. 208 ; A Treatise of Human Nature, ed. by David Fate Norton et Mary J. Norton, (NN), Oxford, Oxford University Press, 2001, 3. 2.7 et 3. 2.8 p. 342-352, trad. de P. Saltel (PS), Paris, G.F., 1993, p. 143-162, Enquiries concerning the Principles of Morals, ed. by Tom L. Beauchamp (TB), Oxford, Clarendon Press, 1998, p. 91, trad. de P. Baranger et P. Saltel (BS), Paris, G.F., 1991, p. 219. Hobbes développe l'hypothèse du contrat social dans le chapitre 17 du Leviathan et Locke dans le chapitre 7 du Deuxième Traité du Gouvernement civil.

${ }^{i i}$ Cf. Reflections on the Revolution in France, ed. O’Brien, Harmondsworth, Penguin Books, 1968, p. 149 sq.

${ }^{\text {iii }}$ Cf. B. Binoche, Les trois sources des philosophies de l'histoire, Paris, P.U.F., 1994, p. 87 sq.

${ }^{\text {iv }}$ Une telle lecture est faite implicitement par B. Stroud, Hume, London, Henley and Boston, Routledge and Kegan Paul, 1977, p. 212, et explicitement par D. Gautier, «David Hume: Contractarian », The Philosophical Review LXXXVIII, n¹, January 1979, p. 3-38.

${ }^{v}$ Cf. D. Miller, Philosophy and Ideology in Hume's Political Thought, New York, Oxford University Press, 1981, p. 13, et P.F. Brownsey, «Hume and the social contract», Philosophical quarterly, vol. 28, n 111, April 1978, Saint Andrews, University Press, W.C. Henderson and son Ltd, p. 132-148.

${ }^{\text {vi }}$ Le point le plus contestable de la présentation humienne réside dans la caractérisation de la morale lockienne comme système égoïste de la morale. Cf. Enquête sur les principes de la morale, appendice 2 (TB p. 91, BS p. 219).

${ }^{\text {vii }}$ Cf. The Letters of David Hume, I, ed. J.Y.T. Greig, Oxford, Clarendon Press, 1932, p. 112.

viii Dialogues concerning Natural Religion, edited with an introduction by S. Tweyman (ST), London and New York, Routledge, 1991, 226 pages, trad. de M. Malherbe (MM), Paris, Vrin, 1997, 254 pages. 
ix Sur l'importance de la notion de convention dans la philosophie humienne, cf. Donald W. Livingston, Hume's Philosophy of Common Life, Chicago, Chicago University Press, 1984, p. 4 et p. 66-75.

${ }^{x}$ « Of the Origin of Government », in The Philosophical Works of David Hume, vol. IV, p. 113-116, trad. de M. Malherbe (MM), in Essais et Traités sur plusieurs sujets, tome I, Paris, Vrin, 1999, p. $97-$ 100.

${ }^{x i}$ Sur ce point, cf. M. P. Thompson, «Hume's Critique of Locke and The Original Contract», $I l$ pensiero politico, Anno X, n², 1977, Firenze, Casa editrice, Léo S. Olschki, p. 199-201. Des penseurs comme Sidney dans les Discourses Concerning Governments ou Tyrrell dans sa Bibliotheca Politica insistaient sur l'évidence historique du contrat tout en se réclamant de Locke et de son analyse normative.

xii Il faut bien noter que dans cet essai, Hume fait tour à tour appel au registre de la fondation et à celui de l'événement pour présenter l'hypothèse contractualiste.

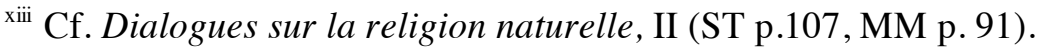

${ }^{\text {xiv }}$ Cf. Dialogues sur la religion naturelle, II (ST p. 109, MM p. 94-95).

${ }^{\mathrm{xv}}$ Enquête sur les principes de la morale, appendice 2 (TB p. 91, BS p. 219). Si cette critique peut sembler injuste eu égard à John Locke dont il semble difficile de faire un partisan de l'hypothèse égoïste (cf. note VI), il faut la lire comme la conséquence de la perspective épistémologique qui soustend l'argumentation de Hume.

${ }^{\text {xvi }}$ Cf. Traité de la nature humaine, 3. 2.2 (NN p. 260, PS p. 87).

${ }^{\text {xvii }}$ Cf. Leviathan, chap. XVII, The Collected Works of Thomas Hobbes, Collected and Edited by Sir William Molesworth, London, Routledge, 1992, vol. III, p. 153 : « The final cause, end, or design of men, who naturally love liberty, and dominion over others, in the introduction of that restraint upon themselves, is the foresight of their own preservation, and of a more contented life thereby ».

xviii J. Terrel, Les théories du pacte social, Paris, Seuil, 2001, p. 249. 


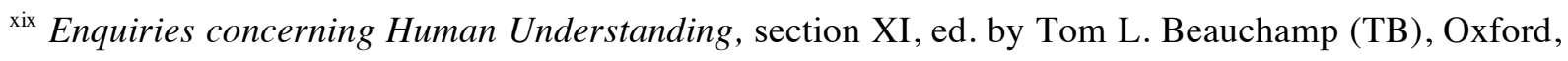
Clarendon Press, 2000, p. 100 sq., trad. de A. Leroy (AL), revue par M. Beyssade, Paris, GF, 1983, p. 211 sq.

${ }^{\mathrm{xx}}$ Enquête sur l'entendement humain, section XI (TB p. 105, AL p. 218).

${ }^{x x i} \ll$ Du contrat originel $($ GG p. 444, FG p. 189).

xxii Par enthousiasme, Hume entend une des deux tendances de la religion populaire, caractérisée par une exaltation de la puissance imaginative et pouvant conduire au fanatisme et à la folie. Hume établit un lien entre l'enthousiasme et le parti Whig. Dans la mesure où les Whigs faisaient référence dans leur doctrine politique à une ancienne constitution fondée sur un contrat originel avant l'invasion de l'Angleterre par les Normands pour s'opposer à la dynastie des Stuart, l'on doit rapprocher le courant contractualiste du parti Whig. Cf. note VII.

xxiii «Of Superstition and Enthousiasm», in The Philosophical Works of David Hume, vol. III, p. 145, trad. de M. Malherbe (MM), in Essais et Traités sur plusieurs sujets, tome I, p. 133.

xxiv « Superstition et enthousiasme » (GG p. 145, MM p. 134).

${ }^{\mathrm{xxv}} \ll$ Du contrat originel » (GG p. 450, FG p. 196).

xxvi « Superstition et enthousiasme » (GG p. 145, MM p. 134).

${ }^{x x v i i}$ Cf. K. Haakonssen, «The structure of Hume's political theory », The Cambridge Companion to Hume, Cambridge, Cambridge University Press, 1993, p. 185, traduction personnelle.

xxviii Traité de la nature humaine, 3. 2.8 (NN p. 347, PS p. 153).

xxix «De la coalition des partis », in The Philosophical Works of David Hume, vol. IV, p. 469, trad. de F. Grandjean, in Discours politiques, p. 217.

${ }^{x x x}$ Dialogues sur la religion naturelle, II (ST p. 110, MM p. 96-97).

${ }^{x x x i}$ Enquête sur l'entendement humain, section XI (TB p. 102-103, AL p. 215).

${ }^{x x x i i}$ Cf. « Du contrat originel » (GG p. 446 et 449, FG p. 192 et 195).

xxxiii Cf. notamment M. Malherbe dans son introduction aux Dialogues sur la religion naturelle, p. 51, F. Brahami, Le travail du scepticisme, Paris, P.U.F., 2001, note 2, p. 171 et M. Groulez, Le scepticisme de Hume : les Dialogues sur la religion naturelle, Paris, P.U.F., 2005, p. 82. 
${ }^{\mathrm{xxxiv}}$ Cf. A Treatise of Human Nature, 1. 4. 1, p. 121, trad. de P. Baranger et P. Saltel (BS), Paris, GF, 1995, p. 262.

${ }^{\mathrm{xxxv}}$ Cf. A Treatise of Human Nature, 2.3. 1, p 260, trad. de J-P Cléro (JPC), Paris, GF, 1991, p. 259 et Enquête sur l'entendement humain, VIII (TB p. 69, AL p. 159-160).

xxxvi Dialogues sur la religion naturelle, IV (ST p. 124, MM p. 121). Notons qu'après avoir examiné ce que la raison pouvait nous dire a priori de l'argument du dessein, Philon présente ce que nous en dit l'expérience. La conclusion est exactement la même : l'ordonnancement de nos pensées apparaît tout aussi délicat et minutieux que celui des végétaux et animaux.

xxxvii « Du contrat originel » (GG p. 455-456, FG p. 202-203).

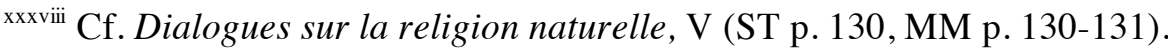

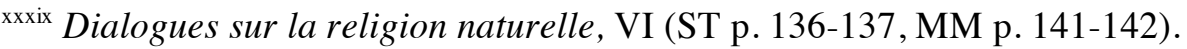

${ }^{x l}$ Il faut noter qu'une telle explication de l'organisation se tient également à l'écart du mécanisme dans la mesure où il y a une adaptation progressive par adaptation aux circonstances.

${ }^{x l i}$ The Natural History of Religion, in The Philosophical Works of David Hume, vol. IV, p. 309 : « As every enquery, which regards religion, is of the utmost importance, there are two questions in particular, which challenge our attention, to wit, that concerning its foundation in reason, and that concerning its origin in human nature », souligné par nous, trad. de M. Malherbe (MM), Paris, Vrin, 1971, p. 39.

xlii Cf. Jennifer Herdt, Religion and faction in Hume's moral philosophy, Cambridge, Cambridge University Press, 1997, p. xii.

xliii Cf. Livingston, Hume's Philosophy of Common Life, p. 60.

xliv «L'origine du gouvernement » (GG p. 113, MM p. 97).

${ }^{\text {xlv }}$ Enquête sur les principes de la morale, section III (TB p. 17, trad. modifiée de BS p. 92-93).

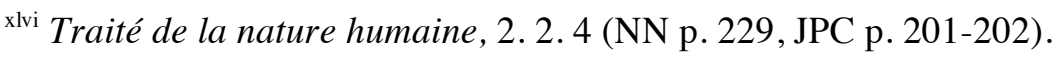

${ }^{\text {xlvii }}$ Traité de la nature humaine, 3. 2.2 (NN p. 312, PS p. 86). 
xlviii Traité de la nature humaine, 3. 2. 2 (NN p. 320, PS p. 99). Annette Baier identifie dans cette perspective, l'amour sexuel, la vie de la famille, et l'amitié comme des conventions primitives, cf. A progress of sentiments, Cambridge, London, Harvard University Press, 1991, p. 228.

${ }^{x}$ lix Traité de la nature humaine, 3.2.2 (NN p. 314, PS p. 89).

${ }^{1}$ Traité de la nature humaine, 3.2.2 (NN p. 314, PS p. 89).

${ }^{\text {li }}$ Cf. Traité de la nature humaine, 3.2.2 (NN p. 313, PS p. 88) et 3. 2.3 (NN p. 323, PS p. 105).

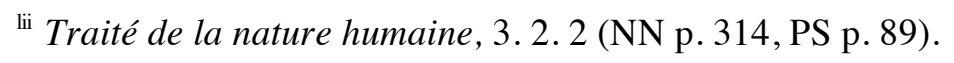

liii Traité de la nature humaine, 3. 2.2 (NN p. 314, PS p. 89).

liv Traité de la nature humaine, 3.2.2 (NN p. 315, trad. modifiée PS p. 90).

${ }^{\text {lv }}$ Cf. Traité de la nature humaine, 2. 1. 11 (NN p. 207, JPC p. 157) et Traité de la nature humaine, 3.3. 1 (NN p. 368, PS p. 197).

${ }^{\text {lvi }}$ Baier Annette, A progress of sentiments, p. 231.

${ }^{\text {lvii }}$ Cf. Traité de la nature humaine, 3.2. 7 (NN p. 345, PS p. 148).

lviii Sur l'importance de l'habitude, cf. «De l'origine du gouvernement» (GG p. 116, MM p. 99), Traité de la nature humaine, 3.2. 10 (NN p. 362, PS p. 182).

lix « Du contrat originel » (GG p. 445-446, FG p. 191).

${ }^{1 x}$ Sur ce point et la typologie des différents niveaux de sympathie, cf. Jennifer Herdt, Religion and faction in Hume's moral philosophy, chap. I et II, p. 17-81. L'auteur accorde une attention particulière au rôle de la sympathie élargie dans la théorie morale pour situer la perspective de Hume par rapport à celle de Hutcheson: Hume se réapproprie la pensée de ce dernier en faisant l'économie de sa dimension apologétique, c'est-à-dire du recours aux causes finales. L'analyse de Herdt déploie ainsi dans le domaine moral ce que nous nous proposons de montrer dans le domaine politique: la sympathie comme principe naturel est le substitut humien au principe de raison.

${ }^{1 x i}$ Traité de la nature humaine, 3. 2.2 (NN p. 320-321, PS p. 101).

${ }^{1 x i i}$ Traité de la nature humaine, 3.3.1 (NN p. 374, PS p. 208).

1xiii Traité de la nature humaine, 3. 3. 1 (NN p. 371-372, PS p. 203-204).

${ }^{\text {lxiv }}$ Traité de la nature humaine, 3. 3.3 (NN p. 384, PS p. 229). 
${ }^{\text {lxv }}$ Cf. E. Durkheim, « La détermination du fait moral», Société et Philosophie, Paris, P.U.F., 2002, p. 92-94. 